\title{
3D automatic segmentation method for retinal optical coherence tomography volume data using boundary surface enhancement
}

\author{
Yankui Sun*, Tian Zhang ${ }^{\dagger}$ and Yue Zhao* \\ Department of Computer Science and Technology \\ Tsinghua University \\ Beijing 100084, P. R. China \\ *syk@mail.tsinghua.edu.cn \\ †kasperlzhang@gmail.com \\ †zhaoyue_82@163.com \\ Yufan He \\ Department of Electronic Engineering \\ Tsinghua University \\ Beijing 100084, P. R. China \\ heyufan1995@qq.com
}

Received 6 July 2015

Accepted 17 September 2015

Published 4 November 2015

\begin{abstract}
With the introduction of spectral-domain optical coherence tomography (SD-OCT), much larger image datasets are routinely acquired compared to what was possible using the previous generation of time-domain OCT. Thus, there is a critical need for the development of three-dimensional (3D) segmentation methods for processing these data. We present here a novel 3D automatic segmentation method for retinal OCT volume data. Briefly, to segment a boundary surface, two OCT volume datasets are obtained by using a 3D smoothing filter and a 3D differential filter. Their linear combination is then calculated to generate new volume data with an enhanced boundary surface, where pixel intensity, boundary position information, and intensity changes on both sides of the boundary surface are used simultaneously. Next, preliminary discrete boundary points are detected from the A-Scans of the volume data. Finally, surface smoothness constraints and a dynamic threshold are applied to obtain a smoothed boundary surface by correcting a small number of error points. Our method can extract retinal layer boundary surfaces sequentially with a decreasing search region of volume data. We performed automatic segmentation on eight human OCT volume datasets acquired from a commercial Spectralis OCT system, where each volume of datasets contains 97 OCT B-Scan images with a resolution of $496 \times 512$ (each B-Scan comprising 512 A-Scans containing 496 pixels); experimental results show that
\end{abstract}

${ }^{*}$ Corresponding author.

This is an Open Access article published by World Scientific Publishing Company. It is distributed under the terms of the Creative Commons Attribution 3.0 (CC-BY) License. Further distribution of this work is permitted, provided the original work is properly cited. 
this method can accurately segment seven layer boundary surfaces in normal as well as some abnormal eyes.

Keywords: Optical coherence tomography; boundary surface enhancement; retinal layer segmentation; OCT volume data.

\section{Introduction}

The retina is a complex organization composed of a transparent layer of tissue. Automatic segmentation algorithms that accurately detect the layer structures in frequency-domain optical coherence tomography (OCT) retinal images are critical for the efficient diagnosis of ocular diseases such as glaucoma, diabetic retinopathy, etc. Many OCT image segmentation methods have been developed to segment the retinal layer boundaries with varying levels of success. Fernandez et al. proposed a method that used a structure tensor combined with complex diffusion filtering to segment seven retinal layer boundaries; ${ }^{1}$ Mujat et al. implemented a method to determine the thickness of the retinal nerve fiber layer (RNFL) from OCT images by segmenting two boundaries using anisotropic noise suppression and deformable splines; ${ }^{2}$ Ishikawa et al. recognized retinal layer positions by peaks and valleys in an A-scan intensity profile by using a mean filter for despeckling, which segmented five layer boundaries; ${ }^{3}$ Chiu et al. presented a segmentation method that used graph theory and dynamic programming to segment seven retinal layers; ${ }^{4}$ This method was later extended for segmentation of mouse retinal layers, ${ }^{5}$ anterior eye images ${ }^{6}$ age-related macular degeneration (AMD) images ${ }^{7}$ and diabetic macular edema images $;^{8}$ Using a similar method, Yang et al. ${ }^{9,10}$ utilized a more complex approach to calculate the weights map of graph-based method, using dualscale gradient information and shortest path search techniques to segment intra-retinal boundaries in OCT images. Yazdanpanah et al. used an active contour approach for the segmentation of rodent retinas; ${ }^{11} \mathrm{~A}$ two-step kernel-based optimization was proposed by Mishra et al. ${ }^{12}$ However, the methods in Refs. 11 and 12 was never tested on OCT datasets of human retinas. Itebeddine et al. proposed a global segmentation algorithm based on using active contours and Markov random fields to segment eight retinal layers. ${ }^{13}$

While the aforementioned methods can be used to segment OCT volume data slice-by-slice, most of them require long processing times, and they do not use the correlation between slices well. Recently, three-dimensional (3D) OCT retinal image segmentation techniques have been developed. Zawadzki et al. proposed segmentation methods using a support vector machine (SVM) and machine learning, ${ }^{14,15}$ which could segment one layer once by manual interaction. Similar to Zawadzki et al. method, a SVM was used to classify pixels in the OCT image but in a fully automated way. ${ }^{16} \mathrm{In}$ Ref. 17, random forest classifier was built to segment eight retinal layers in macular cube images acquired by OCT. The random forest classifier learns the boundary pixels between layers, producing an accurate probability map for each boundary, which is then processed to finalize the boundaries. Kajić et al. proposed a method that used a large training dataset obtained from manual segmentations by human operators as input to develop a statistical model to segment seven retinal layers. ${ }^{18}$ Garvin et al. proposed a graph search-based 3D OCT retinal image segmentation algorithm, ${ }^{19,20}$ which could segment five retinal layers, which was later extended to incorporate hard/soft constraints. ${ }^{21}$ Lee used multi-scale 3D graph search for segmenting the optic nerve head. ${ }^{22}$ Raheleh et al. proposed spectral-geometric methods for graphbased image segmentation and explored a two-step diffusion map approach for the segmentation of OCT images. ${ }^{23}$ Bogunović et al. proposed graphtheoretic method for multi-surface multi-field co-segmentation of intraretinal layers, assuring consistent segmentation of the fields across the overlapped areas. ${ }^{24}$ Shi et al. developed a method to automatically segment the retinal layers in 3D OCT data with serous retinal pigment epithelial detachments (PED), which contains fast denoising and Bscan alignment, multi-resolution graph searchbased surface detection, PED region detection and surface correction above the PED region. ${ }^{25}$ Tian et al. presented a method to segment OCT volume data in the macular region fast and accurately using the shortest path-based graph search. ${ }^{26}$ Fabritius 
et al. presented a fast segmentation method for segmenting the internal limiting membrane (ILM) and the retinal pigment epithelium (RPE) that was based on variations in pixel intensity; ${ }^{27}$ this method used A-Scan to segment 3D OCT images, where only two boundaries are detected. Niu et al. proposed an algorithm to segment 3D OCT images that utilizes a customized edge flow to produce the edge map and a convolution operator to obtain local gradient map in the axial direction. A valid search region is then defined to identify layer boundaries, and a spatial correlation smoothness constraint is applied to remove anomalous points at the layer boundaries. ${ }^{28}$ In Ref. 29, a user-guided segmentation method was proposed to perform the segmentation of retinal layers and features in OCT images. Kafieh et al. investigated 3D data-driven multiscale atomic representation of optical coherence tomography and the applications of complex wavelet-based K-SVD in speckle reduction of OCT datasets and diffusion wavelets in image segmentation. ${ }^{30}$ In Ref. 31 , Chen et al. quantitatively investigated the optical intensity of each retinal layers in central retinal artery occlusion (CRAO), and it found that the inner nuclear layer was identified as the best indicator of CRAO. In Ref. 32, Wang et al. combined the level set method, $k$-means and MRF method to segment three intra-retinal layers around optical nerve head, and they also used hybrid methods such as the level set method, the hysteresis thresholding method and the multi-region continuous max-flow method to segment intra-retinal layers of 3D Macular Images. ${ }^{33}$

In this paper, we propose a novel 3D segmentation method for extracting retinal layer boundaries from OCT volume data using boundary surface enhancement and smoothness surface constraints, which is robust to blood vessel shadow and noise. To segment a boundary surface, two OCT volume datasets are obtained by using a 3D smoothing filter and a 3D differential filter. Their weighted sum is then calculated to generate new volume data with an enhanced boundary surface, where the pixel intensity, boundary position information, and intensity changes on both sides of the boundary surface are used simultaneously. Then, preliminary discrete boundary points are detected from the A-Scans of the volume data. Finally, surface smoothness constraints and a dynamic threshold are applied to obtain a smoothed boundary surface by correcting a small number of error points. Our methods can extract retinal boundary surfaces sequentially within a decreasing region of volume data. The key idea is to use pixel position information, gradient information and intensity information simultaneously to enhance the boundary surface to be detected so that preliminary discrete boundary points can be detected more correctly and error points can be eliminated more easily.

This paper is organized as follows: Section 2 gives a description of our generalized layer segmentation algorithm, which is fundamental for segmenting all of the layer boundary surfaces; Section 3 demonstrates how to segment seven retinal layer boundary surfaces in detail; experimental results and analysis are given in Sec. 4; and conclusions are made in Sec. 5 .

\section{A Generalized Layer Segmentation Algorithm}

An image acquired from a commercial Spectralis OCT device (Heidelberg Engineering, Heidelberg, Germany) is shown in Fig. 1, where the left panel shows the scanning position in the retinal tissue and the right panel shows the corresponding SD-OCT image. Figure 2 illustrates a volume dataset made up of a sequence of SD-OCT B-scans in the $y$ direction. Every B-scan is composed of A-scans in the $x$ direction. Each A-scan has a depth coordinate $z$, which increases going from top to bottom in the image.

A retinal OCT image consists of layer structures (Fig. 3), where the intensity varies in the layers due to differences in the reflection properties of the retinal tissue. Moreover, layer boundaries have various orientations such as the Vitreous-ILM layer boundary, which exhibits a dark layer above a light layer.

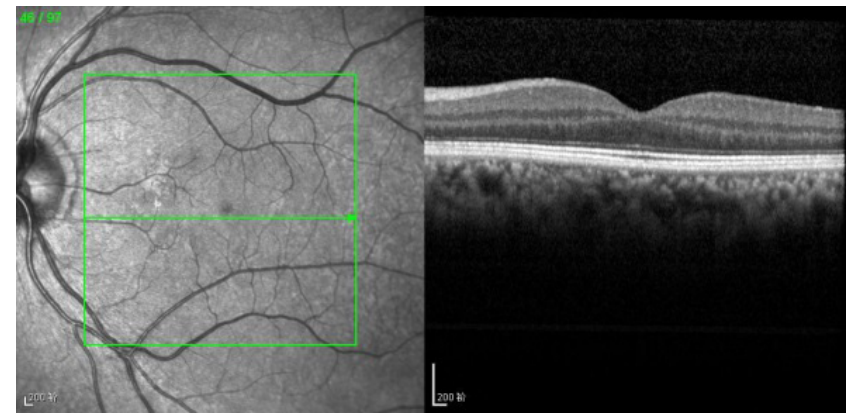

Fig. 1. SD-OCT image. 


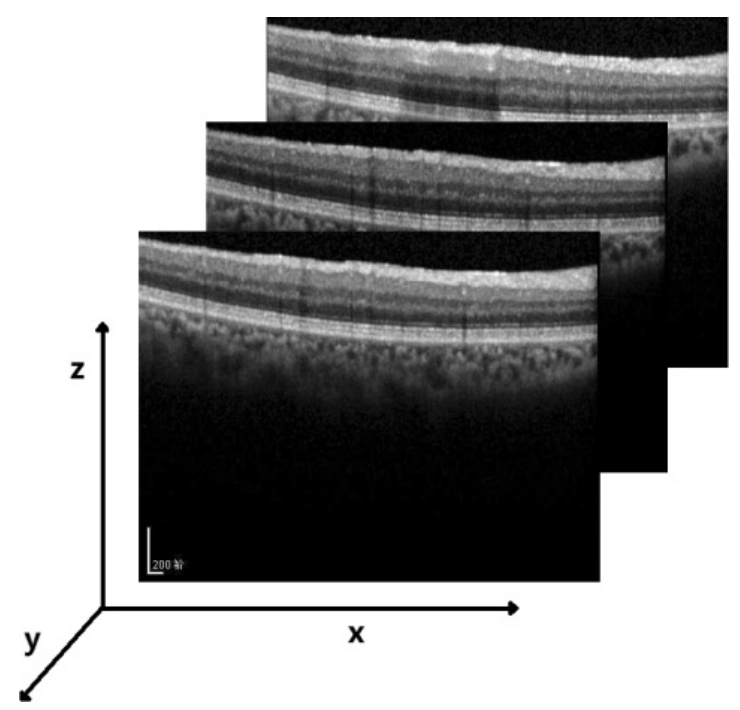

Fig. 2. Illustration of a volume dataset.

This section proposes a method for segmenting the layer structures of the retina. The basic idea of this method is to use the characteristics of the boundary of interest to design a 3D operator and apply it to the original volume data so that the pixel value on the desired boundary in the new volume data is likely to be the maximum value in its A-scan. This approach makes the new volume data a better indicator of the desired boundaries. The algorithm consists of three steps: denoising, extracting boundary points and correcting error points. Obviously, the key problem is to determine how to identify the correct discrete boundary points. Our algorithm tries to achieve accurate boundary point detection by enhancing the boundary of interest. The core steps in our basic retinal layer

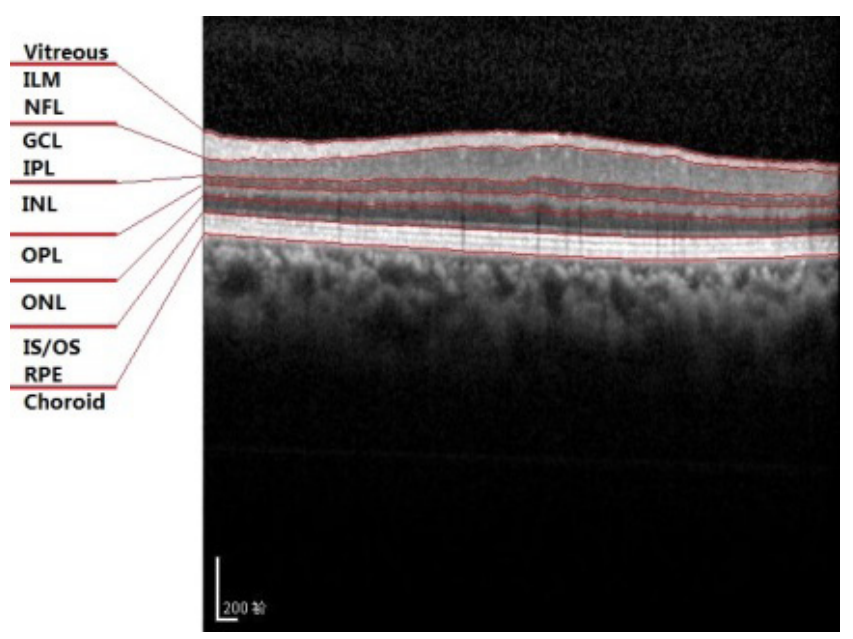

Fig. 3. Retinal OCT image with layer structures. boundary surface segmentation (BRLBSS) algorithm are below.

Step 1: Denoising

A $3 \mathrm{D}$ average filter with size of $K_{1} \times K_{2} \times K_{3}$ is applied to the original retina OCT volume data to obtain a smoothed volume data, $S$, where $K_{1}, K_{2}$ and $K_{3}$ stand for the filter window width in the $x, y$, and $z$ directions, respectively, and are all positive odd numbers.

Step 2: Extract the boundary points

(1) Enhance the boundary with gradient information

A 3D differential filter with a size of $M_{1} \times M_{2} \times$ $M_{3}$ is applied to the original retina OCT volume data to obtain a differential volume data, $D$, where $M_{1}, M_{2}$ and $M_{3}\left(M_{3} \neq 1\right)$ are all positive odd numbers, similar to $K_{1}, K_{2}$ and $K_{3}$.

For an original volume data, $V$, and one boundary of interest to be segmented, a pixel $v_{i_{0}, j_{0}, k_{0}}$ in $V$, is taken as a center point, and a cuboid with size $M_{1} \times M_{2} \times M_{3}$ is constructed using the pixel and its adjacent pixels, $v_{i, j, k}$, where $i, j$, and $k$ are the $x, y$, and $z$ coordinates of each pixel. The differential filter is sensitive to the boundary orientation.

For the RPE-Choroid, OPL-ONL, IPL-INL and NFL-GCL boundaries,

$$
f_{i, j, k}= \begin{cases}1, & k<k_{0} \\ 0, & k=k_{0} \\ -1, & k>k_{0} .\end{cases}
$$

For the Vitreous-ILM, ONL-IS/OS and INL-OPL boundaries,

$$
f_{i, j, k}= \begin{cases}-1, & k<k_{0} \\ 0, & k=k_{0} \\ 1, & k>k_{0} .\end{cases}
$$

Then, the differential filter is used to obtain a differential volume data, $D$. Specifically, for every cuboid in $V$, the intensity sum of the pixels above/ below the cuboid center is subtracted from that of the pixels below/above the cuboid center, and the result is averaged for $M_{1} \cdot M_{2} \cdot\left(M_{3}-1\right)$ to obtain the intensity of the cuboid center.

(2) Enhance the boundary by position, gradient and intensity

The depth, gradient and intensity information for the boundary are used to generate a boundaryenhanced volume data, $I$, to further highlight the 
boundary of interest. $I$ is calculated as follows:

$$
I_{i, j, k}=w_{1} D_{i, j, k}+w_{2} S_{i, j, k},
$$

where $i, j$, and $k$ are the $x, y$, and $z$ coordinates of each pixel, and $w_{1}, w_{2}$ are nonnegative real numbers that are directly proportional to the depth coordinate, $k$.

The intensity of the desired boundary in $I$ is likely to be the maximum value in its A-scan.

\section{(3) Extract the boundary surface points}

In each A-scan of $I$, the point with the highest pixel intensity or the first peak (up to down or down to up, depending on the direction of the boundary of interest) is taken as the preliminary location of the desired boundary. Because the highest pixel intensity value is likely to lie on the desired boundary positions, the results obtained by this approach generally produce an accurate estimate of the location of the boundary.

\section{Step 3: Correct the error points}

The depth positions with large errors are corrected using surface smoothness constraints, which require that the difference between the $z$ coordinates of adjacent pixels is small. In step 2, the boundary surface preliminary positions $(z$ coordinates) make up a depth information matrix, $A$. Given a weighted matrix, $W_{1}$, for a depth element, $p$, in $A$, the absolute value of the difference between $p$ and the weighted average of its adjacent entries is called the error distance (ED) of the element (associated with $W_{1}$ ). For a threshold $T$, if the ED of the element $p$ is larger than the threshold, then $p$ is considered to be an error point (associated with the threshold $T$ ). Given a weighted matrix $W_{2}$, if an element $p$ in $A$ is an error point, the weighted average of its adjacent entries with $W_{2}$ is taken as its correcting value (associated with $W_{2}$ ). The matrix $A$ can be smoothed through iteration as follows:

Choose a number of iterations, $N$. For the $i$ th iteration, pick a threshold, $T_{\mathrm{i}}$. For each entry in $A$, calculate its ED. If an entry is an error point, then it is replaced by its corresponding correcting value. If there exists at least one error point and the iteration $i$ is not equal to $N$, then advance to the next iteration until either there are no error points or $N$ is reached.

The final depth information in $A$ constitutes the desired boundary surface.

\section{Implementation of the Algorithm for Segmenting Seven Retinal Layer Boundary Surfaces}

This section details the implementation of the segmentation algorithm in Sec. 2 that automatically segments seven prominent retinal boundary surfaces in SD-OCT volume data. Figure 4 shows a full schematic of this algorithm, where RPE-Choroid, Vitreous-ILM, IPL-INL, INL-OPL, OPL-ONL, ONL-IS/OS and NFL-GCL boundary surfaces are detected one by one.

For an original OCT volume data $V$, RPEChoroid surface is detected by BRLBSS algorithm in Sec. 3.1, which can deal with retinal SD-OCT images with prominent vessels by using dyadic threshold. Vitreous-ILM surface detection is described in Sec. 3.2. Vitreous-ILM boundary surface segmentation (VI_BSS) algorithm is given to process low noise retinal OCT image. To deal with OCT images with significant noise above the ILM, Vitreous-ILM preliminary boundary surface segmentation (VI_PBSS) algorithm is proposed. VI_BSS is a method without using Gray-scale morphological corrosion, while VI_PBSS uses a ball structure Gray-scale morphological corrosion. Gray-scale morphological corrosion can greatly reduce noise above ILM, but it will also expand the vessel shadow if exists. But without using it, the noise will influence finding ILM. Since VI_PBSS is able to deal with OCT images with prominent vessel shadow, so in order to reduce as much noise as we can as well as maintain the vessel pixels, we merge the boundary points obtained from the VI_BSS and VI_PBSS algorithms to obtain the final segmentation result of Vitreous-ILM boundary surface. Once the RPE-Choroid and Vitreous-ILM boundary surfaces are determined, the middle boundary surfaces between them ONL-IS/OS, OPL-ONL, INL-OPL, IPL-INL, and NFL-GCL can be segmented sequentially moving from down to up in a smaller and smaller search space.

The following subsections discuss each of the outlined steps.

\subsection{RPE-Choroid boundary surface detection}

Based on prior knowledge, the RPE layer is one of the most hyper-reflective layers within a retinal SDOCT image. Thus, the RPE-Choroid boundary 


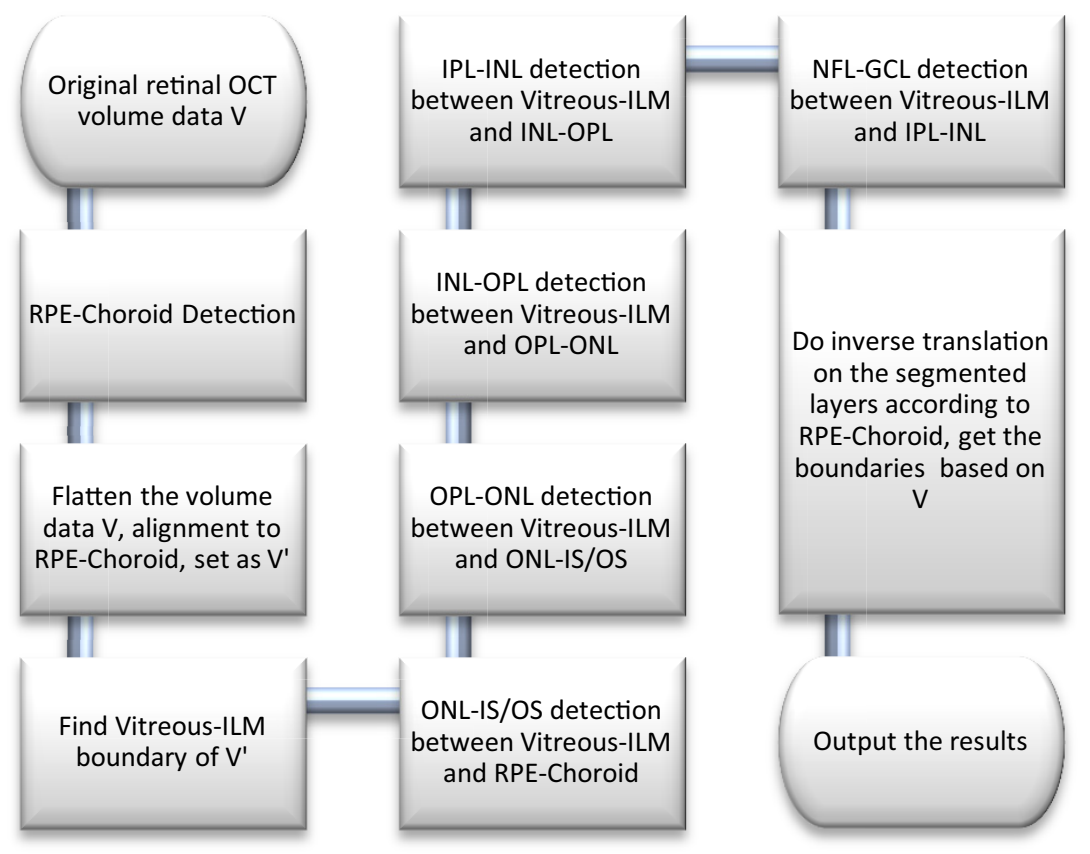

Fig. 4. Seven retinal layer boundary surface segmentation algorithm schematic for SD-OCT volume data.

surface detection is processed first and its implementation is performed according to BRLBSS algorithm in Sec. 2. Here we discuss how to choose the parameters in BRLBSS algorithm.

- The filter size

In a 3D smooth filter $K_{1} \times K_{2} \times K_{3}$ and 3D differential filter $M_{1} \times M_{2} \times M_{3}, K_{1}, K_{2}, K_{3}, M_{1}, M_{2}$ and $M_{3}\left(M_{3} \neq 1\right)$ are all positive odd numbers. In general, cube filter is used with $3 \leq K_{j}, M_{j} \leq 11$ $(i, j=1,2,3)$. These parameters can be adjusted according to dataset processed. A cube filter with a larger size window could much better smooth the data but would spend more computing time. For SD-OCT images with prominent vessels, since RPEChoroid boundary in every B-scan is relatively flat, choosing filters with relatively larger windows can smooth the original data more so as to reduce the influence of vessel containing regions. Our experiments show that it is a good choice to choose $K_{j}=$ $M_{j}=7(j=1,2,3)$ in general, but for OCT images with obvious shadows caused by vessels, it is a better choice to take $K_{j}=M_{j}=11$.

- The weight parameters

In formula (3), the weight parameters $w_{1}, w_{2}$ are nonnegative real numbers that are directly proportional to the depth coordinate, $k$. As an example, we can take $w_{1}=w_{2}=k$.

\section{- Weighted matrices}

In Step 3 in BRLBSS algorithm, two weighted matrices $W_{1}$ and $W_{2}$ are not unique. As an example, we take them as follows.

$$
\begin{gathered}
W_{1}=\frac{1}{138}\left[\begin{array}{ccccccc}
0 & 1 & 1 & 1 & 1 & 1 & 0 \\
1 & 2 & 2 & 2 & 2 & 2 & 1 \\
2 & 4 & 4 & 4 & 4 & 4 & 2 \\
4 & 8 & 16 & -138 & 16 & 8 & 4 \\
2 & 4 & 4 & 4 & 4 & 4 & 2 \\
1 & 2 & 2 & 2 & 2 & 2 & 1 \\
0 & 1 & 1 & 1 & 1 & 1 & 0
\end{array}\right], \\
W_{2}=\frac{1}{138}\left[\begin{array}{ccccccc}
0 & 1 & 1 & 1 & 1 & 1 & 0 \\
1 & 2 & 2 & 2 & 2 & 2 & 1 \\
2 & 4 & 4 & 4 & 4 & 4 & 2 \\
4 & 8 & 16 & 0 & 16 & 8 & 4 \\
2 & 4 & 4 & 4 & 4 & 4 & 2 \\
1 & 2 & 2 & 2 & 2 & 2 & 1 \\
0 & 1 & 1 & 1 & 1 & 1 & 0
\end{array}\right]
\end{gathered}
$$

- Iterations and thresholds

For smoothing, iteration times and thresholds should be determined reasonably. The more iterations, the more computing time. In order to deal with OCT images with prominent vessels, dynamic thresholds are proposed and used here. Dynamic threshold decreases as iterations increase. These parameters can be adjusted on different datasets. 


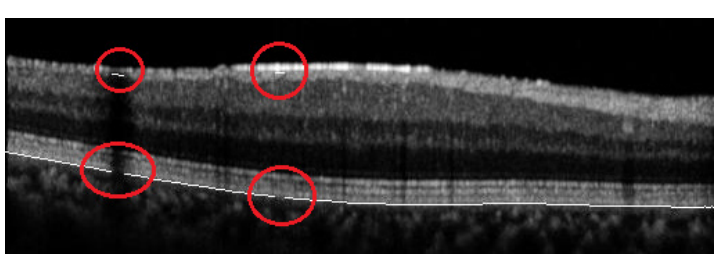

Fig. 5. Great outlier points exist before smoothness.

Here some empirical values are provided according to the test datasets. We choose the number of iterations $N=25$. For the first 20 iterations, a dynamic threshold is used according to $T_{i}=$ count $\times$ $i / 2000$, where count is the element number in matrix $A, i$ is the iteration number and 2000 is an empirical value. In the last 5 iterations, the threshold is fixed at 1. Experimental results in Figs. 5-7 illustrate that dyadic thresholds can quickly and accurately correct outlier points. Figure 5 shows the existence of outlier points before smoothing.

Figure 6 shows the processed result for Fig. 5 after five iterations using a dynamic threshold. It can be seen that the outlier points approach correct points. Figure 7 shows the final smoothed result after 20 iterations.

Some retinal layers may have large curvature in SD-OCT images, such as fovea region. To correct for curvature, we flatten the images to enhance their adaptation for the segmentation algorithm. In this process, the image below the RPE-Choroid boundary is ignored. Figure 8 demonstrates retinal

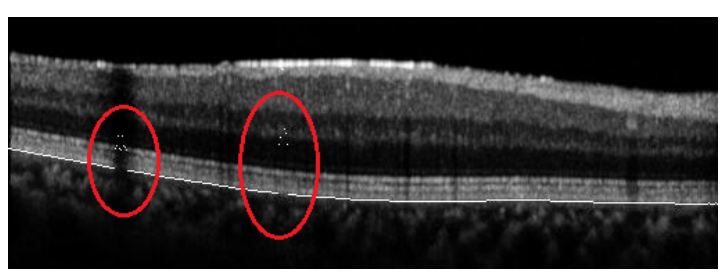

Fig. 6. The processed result of Fig. 5 after five iterations by using dynamic threshold.

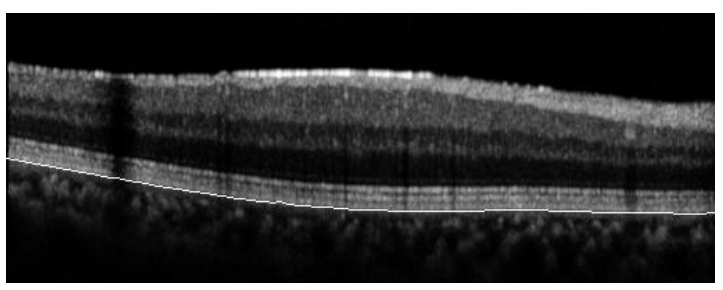

Fig. 7. The final smoothed result after 20 iterations.

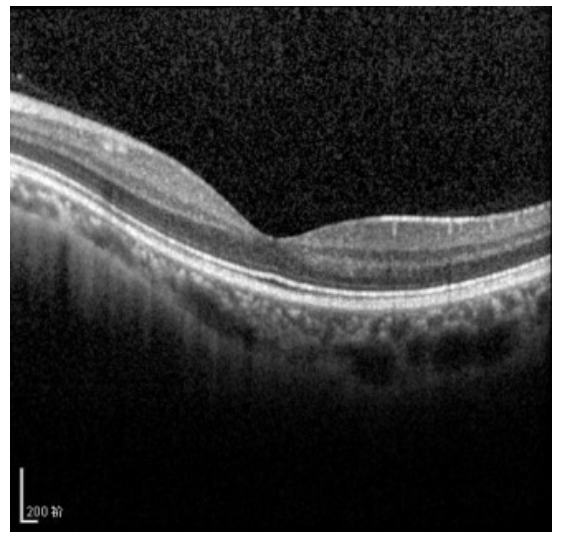

(a)

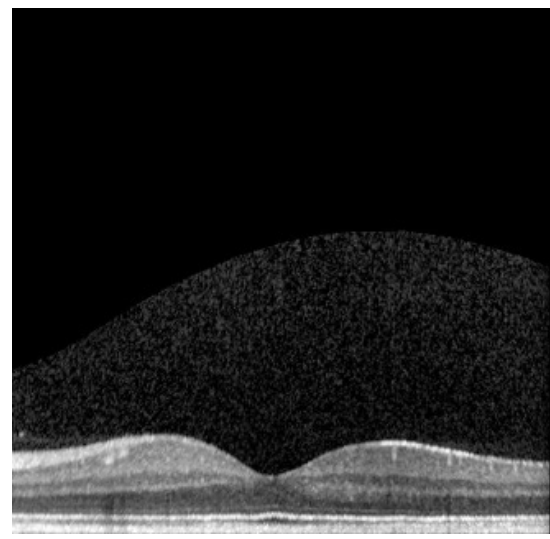

(b)

Fig. 8. Image flattening. (a) The original retinal SD-OCT image. (b) The flattened image without the region below the RPE-Choroid boundary.

flattening, where Fig. 8(b) is the flattened version of the original image, Fig. 8(a).

\subsection{Vitreous-ILM boundary surface segmentation}

Vitreous-ILM boundary surface detection is processed on the flattened volume data of the original volume data $V$ by ignoring the pixels below the RPE-Choroid boundary surface. For a low noise retinal OCT image where only background noise exists above the ILM, similar to the RPE-Choroid boundary surface detection, the BRLBSS algorithm in Sec. 2 can be used to obtain an accurate segmentation result. However, because the VitreousILM boundary may have a large curvature in the fovea region, the choice of the related parameters should be considered carefully. For example, 
relatively narrow $3 \mathrm{D}$ filters in the $x$ direction (Fig. 2) should be used for the fovea region.

\section{Vitreous-ILM boundary surface segmenta-} tion (VI_BSS) algorithm:

\section{Step 1: Denoising}

For the flattened volume data $V^{\prime}$, a $3 \mathrm{D}$ average filter with window size $6 \times 6 \times 6$ is used to produce a volume data denoted $M$. Then, a threshold filter is applied to $M$. In our experiment, the threshold value was 30 . This filter procedure was iterated several times to obtain a smoothed volume data, $S$.

Step 2: Extract boundary points

(1) Enhance the boundary with gradient information

A differential filter in the $z$ direction is applied to the volume data $S$ using a cuboid with a size of $1 \times 1 \times 11$. The new volume data is denoted by $D$.

\section{(2) Extract discrete boundary points}

In every A-Scan of the volume data $D$, the first peak point is determined by moving from up to down. These points constitute the preliminary VitreousILM boundary surface.

\section{Step 3: Correct error points}

This step is similar to the RPE-Choroid boundary surface smoothness scheme. The main difference is the smaller size of the weighted matrices that are used to adapt the large curvature features of ILM layer in the fovea region. The weighted matrices are:

$$
\begin{gathered}
W_{1}=\frac{1}{64}\left[\begin{array}{ccccc}
0 & 1 & 2 & 1 & 0 \\
1 & 4 & 8 & 4 & 1 \\
2 & 8 & -64 & 8 & 2 \\
1 & 4 & 8 & 4 & 1 \\
0 & 1 & 2 & 1 & 0
\end{array}\right], \\
W_{2}=\frac{1}{64}\left[\begin{array}{lllll}
0 & 1 & 2 & 1 & 0 \\
1 & 4 & 8 & 4 & 1 \\
2 & 8 & 0 & 8 & 2 \\
1 & 4 & 8 & 4 & 1 \\
0 & 1 & 2 & 1 & 0
\end{array}\right] .
\end{gathered}
$$

In the last five iterations, the size of weighted matrices is reduced further. They are:

$$
W_{1}=\frac{1}{12}\left[\begin{array}{ccc}
1 & 2 & 1 \\
2 & -12 & 2 \\
1 & 2 & 2
\end{array}\right], \quad W_{2}=\frac{1}{12}\left[\begin{array}{lll}
1 & 2 & 1 \\
2 & 0 & 2 \\
1 & 2 & 2
\end{array}\right]
$$

The VI_BSS algorithm is effective for OCT images with vessel shadows and/or a fovea region.

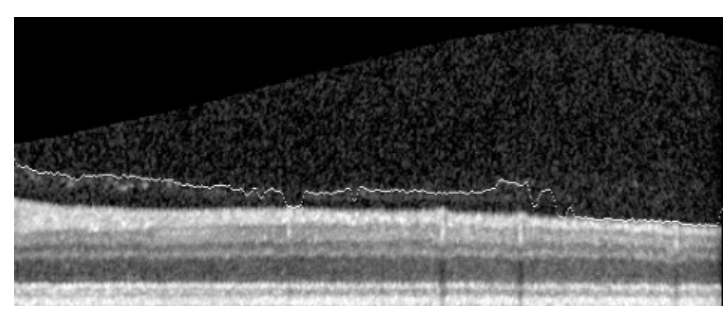

Fig. 9. Incorrect segmentation because of serious noise above ILM.

However, it may fail for clinical OCT images with significant noise above the ILM (Fig. 9). To adapt this case, an improved algorithm is proposed.

Vitreous-ILM preliminary boundary surface segmentation (VI_PBSS) algorithm:

Step 1: Denoising

Gray-scale morphological corrosion with a ball structure of radius $r$ is performed on the smoothed volume data $S$ obtained from the VI_BSS algorithm. Then, the average and threshold filter processing is repeated, as done in Step 1 of the VI_BSS algorithm. We denote the denoised volume data with $S E$. In our implementation, $r=5$ was used. Figure 10 illustrated the denoising effect.

Step 2: Extract the preliminary boundary points (1) Enhance the boundary with gradient information.

A differential filter in the $z$ direction is applied to the volume data $S E$ using a cuboid with a size of $1 \times 1 \times 11$. The new volume data is denoted by $D$.

\section{(2) Extract discrete boundary points}

In every A-Scan of the volume data $D$, determine the first peak point moving from up to down. To compensate for the excessive erosion, $\lceil r / 2\rceil$ pixels are subtracted from the depth coordinate, and these points constitute the preliminary Vitreous-ILM boundary surface points.

The VI_PBSS algorithm works well for OCT images with considerable noise above the ILM, but it may fail for clinical OCT images with prominent vessels (Fig. 11) due to excessive erosion.

Next, we merge the boundary points obtained from the VI_BSS and VI_PBSS algorithms to obtain the final segmentation result. The basic strategy is to replace the discontinuous boundary points obtained from the VI_PBSS algorithm with the corresponding boundary points obtained from the VI_BSS algorithm. 


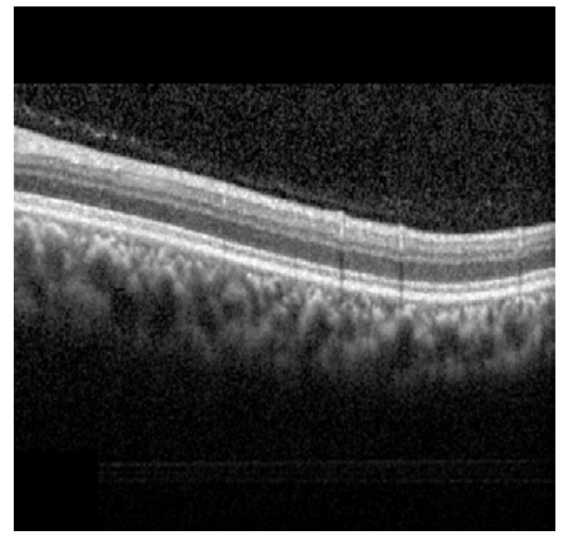

(a)

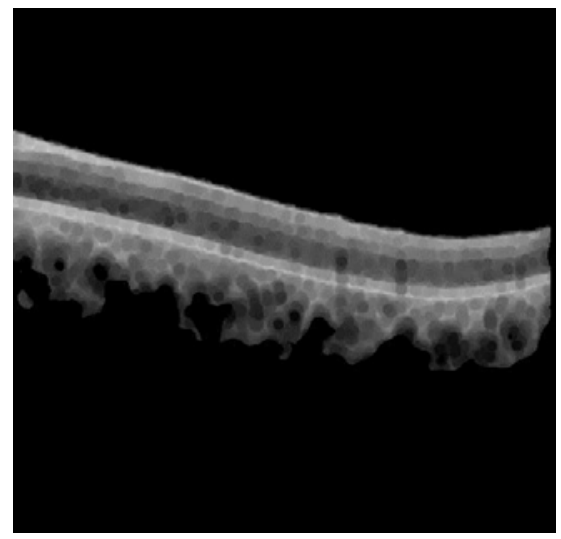

(b)

Fig. 10. Denoising effect using VI_BSS algorithm. (a) Original image. (b) the denoised image using Gray-scale morphological corrosion.

Let $A$ and $B$ be the boundary depth matrices obtained from the VI_PBSS and VI_BSS algorithms, respectively. Matrix $C$ is the average filter result with a window size of $n \times n$ on the boundary depth matrix $A$. In our implementation, $n=11$ was used. Matrix $E$ is the merged boundary depth matrix. If $\left|a_{i j}-c_{i j}\right|<\left|b_{i j}-c_{i j}\right|$, then $e_{i j}=a_{i j}$; otherwise $e_{i j}=b_{i j}$. Finally, the depth matrix $E$ is further smoothed according to step 3 used in the VI_BSS algorithm to obtain the Vitreous-ILM boundary

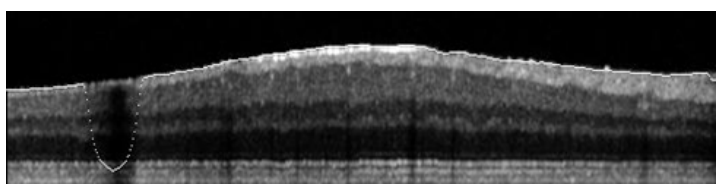

Fig. 11. Incorrect segmentation results because of excessive erosion in a vessel or shadow region.

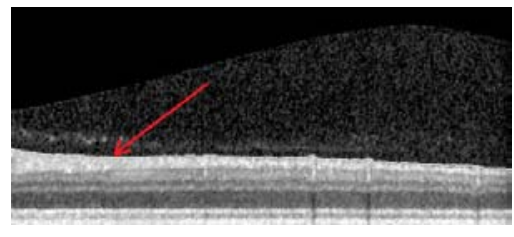

(a)

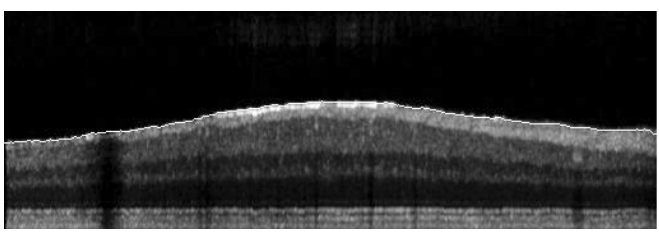

(b)

Fig. 12. Segmentation results with the improved algorithm. (a) Image with considerable noise. (b) Image with a large shadow.

surface segmentation result. Figure 12 shows the segmentation results with the improved algorithm.

\subsection{ONL-IS/OS boundary surface segmentation}

ONL-IS/OS boundary surface segmentation can be done with the flattened volume data $V$ in between the RPE-Choroid and Vitreous-ILM boundary surfaces, where pixel intensities are set to 0 below the RPE-Choroid boundary and to 255 above the Vitreous-ILM boundary. We apply the 3D differential filter in Eq. (2) with a size of $3 \times 3 \times 11$ on the flattened volume data to obtain new volume data, D. In Eq. (3), $w_{1}=k$ and $w_{2}=0$ are used to obtain an enhanced volume data, $I$. In every A-scan of data $I$, the point with the largest pixel value is taken as the preliminary location of the desired boundary. In principle, the ONL-IS/OS boundary surface can be obtained from step 3 in the BRLBSS algorithm. However, this algorithm may not work well for some OCT images with dark spots in the fovea region and near RPE, as shown in Fig. 13.

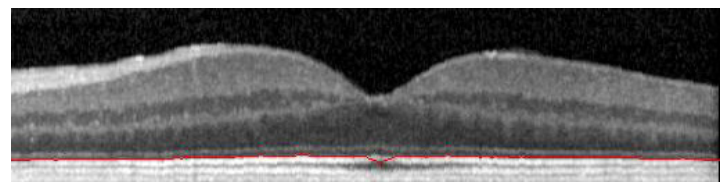

Fig. 13. Incorrect segmentation result in the fovea region and near the RPE because of dark spots. 
To adapt our algorithm to this case, another error correction procedure can be added before step 3 in the BRLBSS algorithm. Because the IS/OS layer is relatively flat, a third order polynomial can be applied to rule out some of the error points. This procedure works as follows:

(1) For the preliminary boundary points of each B-scan in $I$, polynomial least squares estimates is performed to obtain a fitted polynomial

$$
z=a_{0}+a_{1} x+a_{2} x^{2}+a_{3} x^{3} .
$$

(2) The depth value of every boundary point is substituted into the above expression to obtain a polynomial fitted value. If the depth value is outside of the confidence interval for the estimated value associated with a probability of 0.98 , the boundary point is considered to be a noise point and is eliminated. The remaining credible boundary points are used to repeat the polynomial least squares estimate and obtain another fitted polynomial

$$
z_{1}=a_{4}+a_{5} x+a_{6} x^{2}+a_{7} x^{3} .
$$

(3) The $x$ coordinate of every noise point is substituted into the polynomial $z_{1}$, and the polynomial fitted value is taken as a depth estimate for the noise boundary point.

With the last obtained preliminary ONL-IS/OS boundary points, the error points are corrected using the same algorithm that is used for the RPEChoroid smoothing.

The ONL-IS/OS boundary surface can be segmented correctly by using a polynomial fitting to eliminate the error points (Fig. 14).

\subsection{OPL-ONL, NFL-GCL, IPL-INL, INL-OPL boundary surface segmentations}

To segment the OPL-ONL boundary surface, we further shrink the search space in between the

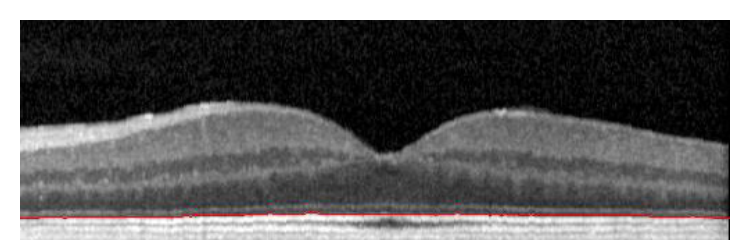

Fig. 14. ONL-IS/OS boundary surface segmentation result obtained by using polynomial fitting to eliminate the error points.
ONL-IS/OS and Vitreous-ILM layer boundaries. The basic steps are similar to the steps used in the ONL-IS/OS boundary segmentation. The differences are as follows:

(1) The OPL-ONL boundary does not appear dark spots like the ONL-IS/OS boundary, and it may have a large curvature when the fovea region lies in the image. As a result, the step using a third-order polynomial to eliminate some error points is not used here.

(2) The 3D differential filter in Eq. (1) with size of $7 \times 15 \times 15$ on the flattened volume data is used.

The other boundary detection algorithms for the NFL-GCL, IPL-INL, and INL-OPL are similar to that used for OPL-ONL boundary detection. When the Vitreous-ILM, ONL-IS/OS, OPL-ONL, NFLGCL, IPL-INL, and INL-OPL segmentations are all completed with the flattened volume data, an inverse translation on the segmented layers is performed according to the RPE-Choroid boundary to obtain the boundaries based on the original volume data $V$.

\section{Experimental Results and Analysis}

To determine the segmentation accuracy of our algorithms for SD-OCT volume data, we performed segmentation on eight OCT volume datasets acquired from a commercial Spectralis OCT device, using Matlab R2012b (The Mathworks, Inc., Natick, MA, USA). In each volume of data, the number of pixels are 512 in the $x$ direction, 97 in the $y$ direction and 496 in the $z$ direction. The pixel spacing in the $x$ direction is $11.55 \mu \mathrm{m}$, in the $y$ direction $61.51 \mu \mathrm{m}$ and $3.87 \mu \mathrm{m}$ in the $z$ direction. The data is saved in AVI video format, and the OCT B-Scan images were first extracted from the AVI file.

Figure 15 shows two images and Vitreous-ILM boundary segmentation results before/after denoising, with the left images in Fig. 15(a), Fig. 15(c) as the segmentation results before denoising, the right images in Fig. 15(b), Fig. 15(d) as the segmentation results after denoising with Gray-scale morphological corrosion. We can see from Fig. 15 that the proposed method is robust to noise.

Figure 16 displays OCT volume datasets obtained by using a 3D smoothing filter, a 3D differential filter and RPE-Choroid boundary surface 


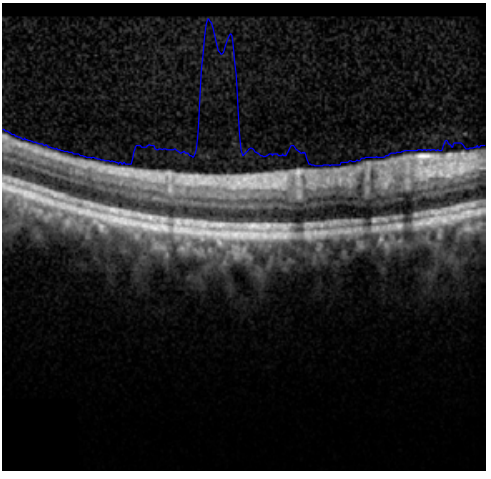

(a)

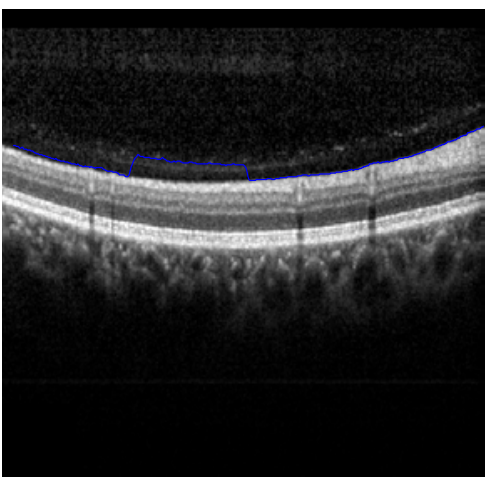

(c)

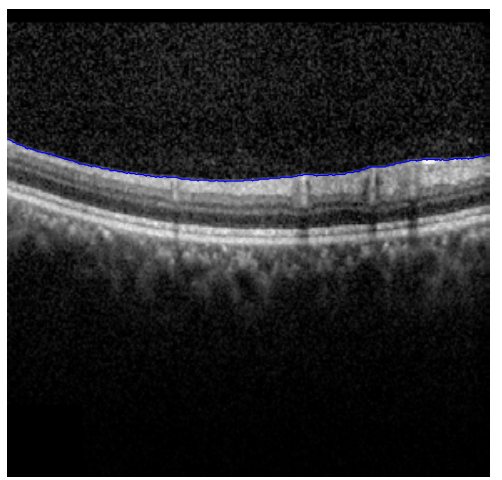

(b)

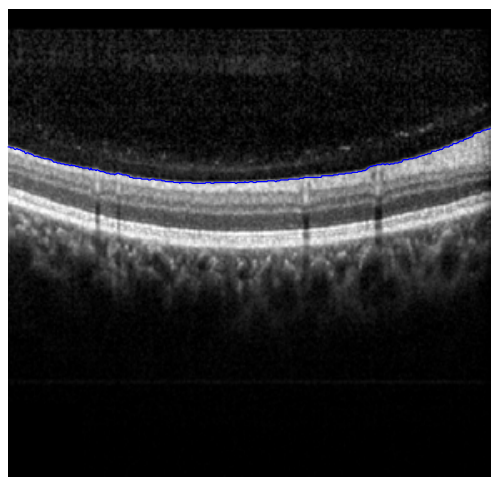

(d)

Fig. 15. Vitreous-ILM boundary segmentation results. (a), (c) before denoising. (b), (d) after denoising with Gray-scale morphological corrosion.

enhancement, with Fig. 16(a) as the original image; Fig. 16(b) as the result of 3D smoothing filter; Fig. 16(c) as the result of 3D differential filter; Fig. 16(d) as the result of adding Fig. 16(b) and Fig. 16(c) together without depth enhancement; Fig. 16(e) as the result of adding Fig. 16(b) and
Fig. 16(c) together with $z$-depth enhancement where the pixel intensity values in the upper part of the RPE-Choroid boundary are weakened so that the RPE-Choroid boundary is enhanced.

Figure 17 illustrates segmentation of seven layer surface boundaries with prominent vessel shadows.

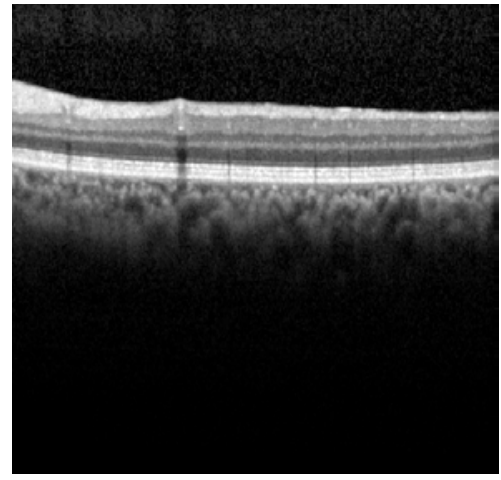

(a)

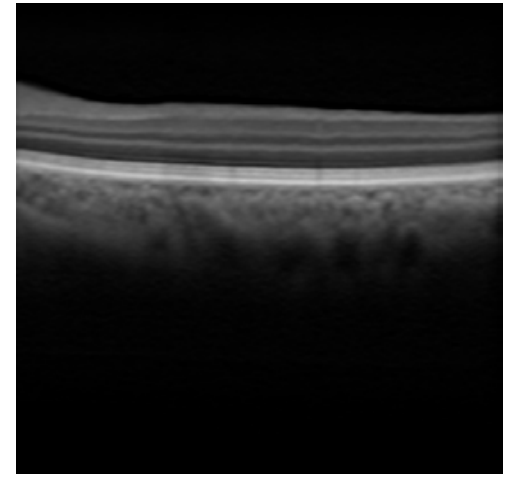

(b)

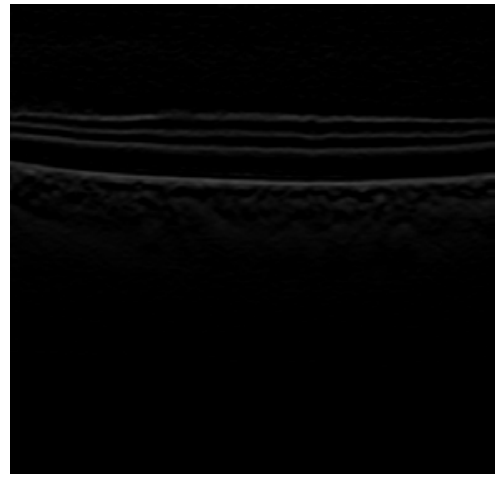

(c)

Fig. 16. OCT volume datasets obtained by using a 3D smoothing filter, a 3D differential filter and RPE-Choroid boundary surface enhancement. (a) the original image. (b) the result of 3D smoothing filter. (c) the result of 3D differential filter. (d) the result of adding (b) and (c) together without depth enhancement. (e) the result of adding (b) and (c) together with $z$-depth enhancement. 


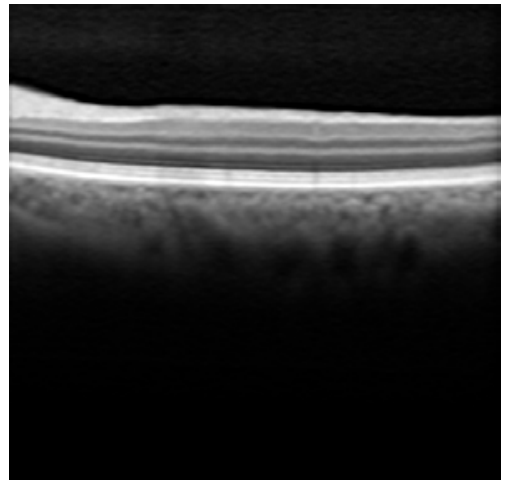

(d)

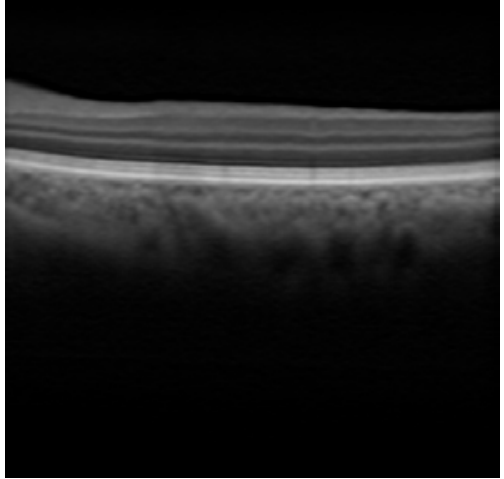

(e)

Fig. 16. (Continued)
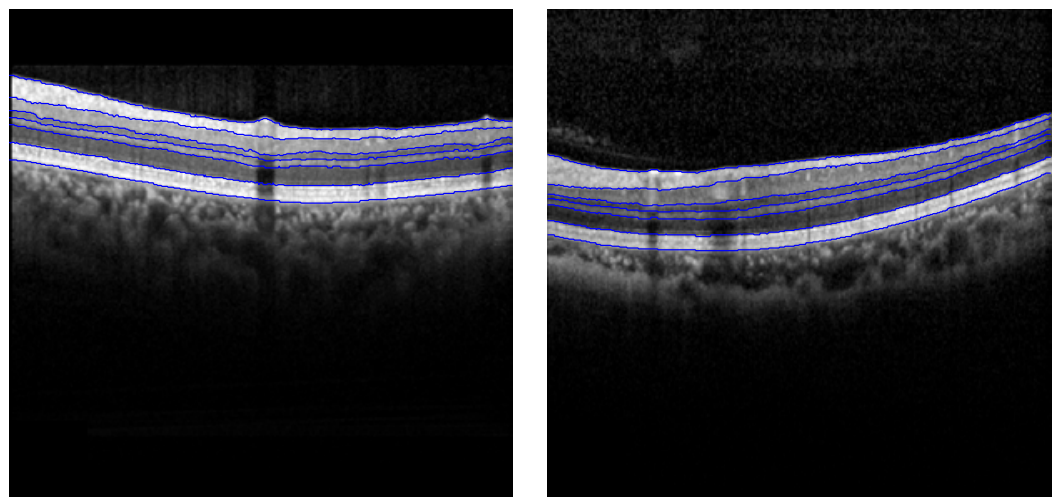

Fig. 17. Segmentation of seven layer surface boundaries with prominent vessel shadows.

To validate the performance of the proposed method, two manual segmentations and the automatic segmentation made by our algorithm are done and their experimental results are compared. We manually delineated an image frame using the internally developed matlab GUI. We choose ten frames randomly from each OCT volume datasets, and delineated seven boundaries manually on each frame. The manual delineations were performed by clicking on approximately $20-50$ points along each layer border followed by interpolation between the points using a cubic B-spline. Segmentation results of some frames are illustrated in Fig. 18, where the manually delineated boundary and the
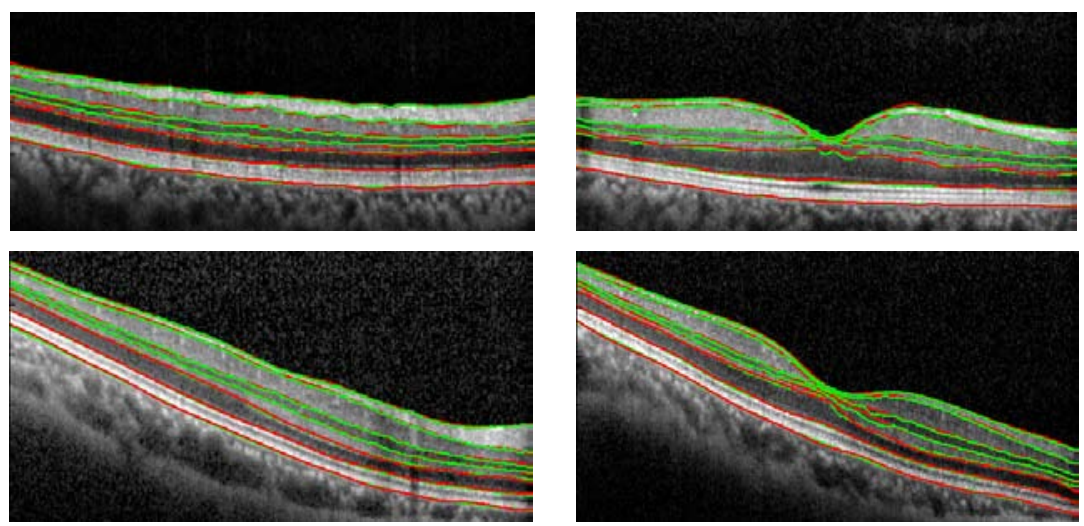

Fig. 18. Segmentation results of some frames. 
Table 1. Mean absolute error and signed errors (and standard deviations) in micron.

\begin{tabular}{|c|c|c|c|c|c|c|}
\hline \multirow[b]{2}{*}{ Boundary } & \multicolumn{2}{|c|}{ Auto vs Exp. 1} & \multicolumn{2}{|c|}{ Auto vs Exp. 2} & \multicolumn{2}{|c|}{ Auto vs avg. Exp } \\
\hline & Absolute & Signed & Absolute & Signed & Absolute & Signed \\
\hline Vitreous-ILM & $4.12(3.32)$ & $2.47(4.24)$ & $2.93(2.72)$ & $-0.50(3.91)$ & $2.75(2.56)$ & $0.97(3.42)$ \\
\hline NFL-IPL & $4.90(5.03)$ & $-1.12(6.47)$ & $3.21(2.57)$ & $-1.17(3.83)$ & $3.26(3.02)$ & $-1.15(4.04)$ \\
\hline IPL-INL & $4.69(4.40)$ & $-0.39(5.74)$ & $3.05(2.33)$ & $-0.68(3.62)$ & $3.09(2.70)$ & $-0.58(3.76)$ \\
\hline INL-OPL & $5.07(4.35)$ & $-3.19(5.46)$ & $3.04(2.27)$ & $-1.59(3.35)$ & $3.47(2.77)$ & $-2.38(3.53)$ \\
\hline OPL-ONL & $4.06(3.27)$ & $-1.04(4.45)$ & $2.95(2.19)$ & $-1.11(3.35)$ & $2.84(2.17)$ & $-1.08(3.08)$ \\
\hline ONL-IS/OS & $2.93(2.13)$ & $1.08(3.06)$ & $2.74(2.04)$ & $-0.20(3.22)$ & $2.24(1.67)$ & $0.46(2.55)$ \\
\hline RPE-Choroid & $3.30(2.43)$ & $0.09(3.54)$ & $2.95(2.15)$ & $-1.39(3.21)$ & $2.34(1.71)$ & $-0.69(2.59)$ \\
\hline Overall & $4.15(3.56)$ & $-0.30(4.71)$ & $2.98(2.32)$ & $-0.95(3.50)$ & $2.85(2.37)$ & $-0.64(3.28)$ \\
\hline
\end{tabular}

Table 2. Avg. manual thickness and auto-segment thickness in micron.

\begin{tabular}{lccc}
\hline Layers & $\begin{array}{c}\text { Manual } \\
\text { thickness }(\mu \mathrm{m})\end{array}$ & $\begin{array}{c}\text { Auto-segment } \\
\text { thickness }(\mu \mathrm{m})\end{array}$ & $\begin{array}{c}\text { Absolute } \\
\text { error }(\mu \mathrm{m})\end{array}$ \\
\hline NFL & 47.23 & 41.20 & 6.03 \\
GCL & 63.84 & 65.05 & 1.21 \\
INL & 28.09 & 26.58 & 1.51 \\
OPL & 30.05 & 31.00 & 0.95 \\
ONL & 67.50 & 68.96 & 1.47 \\
RPE & 64.81 & 60.97 & 3.85 \\
\hline
\end{tabular}

automatically segmented boundary are plotted in the red line and green line respectively. When one color covers the other, this means our algorithm fits well with the manual method at that position. The pictures show that our algorithm is quite accurate no matter whether an image has a fovea or an incline.

The absolute and signed errors for each boundary and their averages are calculated. Table 1 shows that the average absolute error compared to the average manual delineation is $2.85 \mu \mathrm{m}$. To further examine our algorithm, we calculated the thickness of the OCT layers from manual delineated data and auto-segmentation data. The experimental results are given in Table 2. The results listed in Tables 1 and 2 are very similar in essential, which shows that our methods are rather practical for substituting the manual segmentation method.

In order to ensure the accuracy of the proposed segmentation method, we further used OCTSeg to process our dataset, and we compared the results with our manual segmentation results numerically. OCTSeg Software is open for segmenting and visualizing OCT data, which supported Heidelberg Engineering Spectralis RAW data files, Images and image lists, including .vol, .oct, .list, .pgm, .tif, and . jpg format. OCTSeg Software is available at http://www5.cs.fau.de/our-team/mayer-markus/ automated-retinal-layer-segmentation/. It can segment six prominent boundaries, including RPEChoroid, Vitreous-ILM, IPL-INL, OPL-ONL, ONL-IS/OS and NFL-GCL. Our test data is also from Heidelberg Engineering Spectralis, and the proposed algorithm can segment seven prominent

Table 3. OCTSeg mean absolute error and signed errors (and standard deviations) in micron.

\begin{tabular}{|c|c|c|c|c|c|c|}
\hline \multirow[b]{2}{*}{ Boundary } & \multicolumn{2}{|c|}{ Auto vs Exp. 1} & \multicolumn{2}{|c|}{ Auto vs Exp. 2} & \multicolumn{2}{|c|}{ Auto vs avg. Exp } \\
\hline & Absolute & Signed & Absolute & Signed & Absolute & Signed \\
\hline Vitreous-ILM & $4.21(5.77)$ & $1.28(6.98)$ & $5.30(6.13)$ & $4.25(6.98)$ & $4.03(5.58)$ & $2.74(6.41)$ \\
\hline NFL-IPL & $16.41(16.48)$ & $11.00(18.82)$ & $16.21(15.53)$ & $11.20(17.60)$ & $15.81(15.71)$ & $11.05(17.71)$ \\
\hline IPL-INL & $9.39(8.89)$ & $5.66(10.64)$ & $8.96(7.81)$ & $6.01(9.24)$ & $8.55(7.84)$ & $5.82(9.24)$ \\
\hline INL-OPL & $31.20(8.19)$ & $30.97(8.37)$ & $29.89(8.18)$ & $29.65(8.42)$ & $30.60(7.49)$ & $30.41(7.71)$ \\
\hline OPL-ONL & $6.88(5.59)$ & $0.43(7.28)$ & $6.63(5.72)$ & $0.42(7.32)$ & $6.28(5.34)$ & $0.37(6.74)$ \\
\hline ONL-IS/OS & $6.32(4.70)$ & $-3.95(5.87)$ & $6.19(4.56)$ & $-2.63(5.55)$ & $5.72(4.27)$ & $-3.28(5.20)$ \\
\hline RPE-Choroid & $3.21(2.44)$ & $0.18(3.62)$ & $4.00(2.82)$ & $1.65(3.82)$ & $2.99(2.23)$ & $0.94(3.02)$ \\
\hline Overall & $11.09(7.43)$ & $6.51(8.80)$ & $11.03(7.25)$ & $7.22(8.42)$ & $10.57(6.92)$ & $6.86(8.00)$ \\
\hline
\end{tabular}



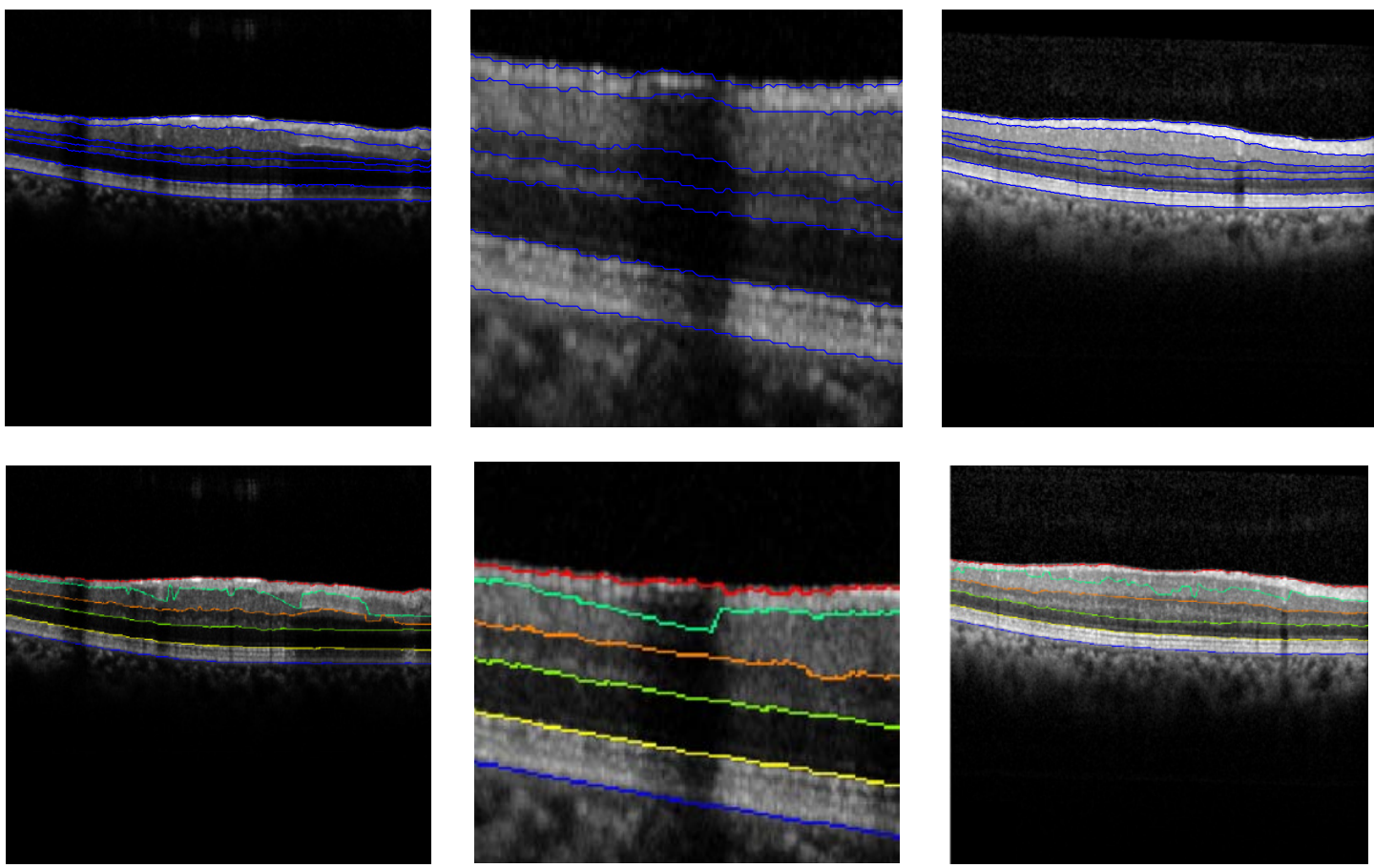

Fig. 19. Comparison with OCTSeg. Our results (first row) and OCTSeg results (the second row).

Table 4. Mean absolute error and signed errors for odd and even B-Scan groups in micron.

\begin{tabular}{lccr}
\hline & \multicolumn{2}{c}{ Auto vs avg. Exp } & \\
\cline { 2 - 3 } Boundary & Odd & Even & \multicolumn{1}{c}{$\begin{array}{c}\text { Difference } \\
\text { Odd - Even }\end{array}$} \\
\hline Vitreous-ILM & $2.71(2.10)$ & $2.80(2.76)$ & $-0.09(-0.66)$ \\
NFL-IPL & $2.98(2.69)$ & $3.43(3.11)$ & $-0.45(-0.42)$ \\
IPL-INL & $2.87(2.52)$ & $3.13(2.64)$ & $-0.25(-0.12)$ \\
INL-OPL & $3.36(2.60)$ & $3.56(2.69)$ & $-0.19(-0.09)$ \\
OPL-ONL & $2.47(1.95)$ & $3.07(2.38)$ & $-0.59(-0.43)$ \\
ONL-IS/OS & $2.29(1.67)$ & $2.25(1.71)$ & $0.04(-0.04)$ \\
RPE-Choroid & $2.16(1.63)$ & $2.54(1.84)$ & $-0.37(-0.21)$ \\
Overall & $2.70(2.17)$ & $2.97(2.45)$ & $-0.27(-0.28)$ \\
\hline
\end{tabular}

boundaries, including RPE-Choroid, Vitreous-ILM, IPL-INL, INL-OPL, OPL-ONL, ONL-IS/OS and NFL-GCL. Compared with OCTSeg, our algorithm can segment more one boundary OPL-ONL than OCTSEg does. OCTSeg can segment our dataset effectively as a whole, so we compare our method with OCTSeg using our datasets. The absolute error and signed error is shown in Table 3.

From Tables 1 and 3, we can see that our algorithm works better at every boundary. The OCTSeg fails to segment NFL-IPL and INL-OPL correctly but works well on Vitreous-ILM and RPE-Choroid. However, our algorithm outperforms OCTSeg greatly in all these boundaries.

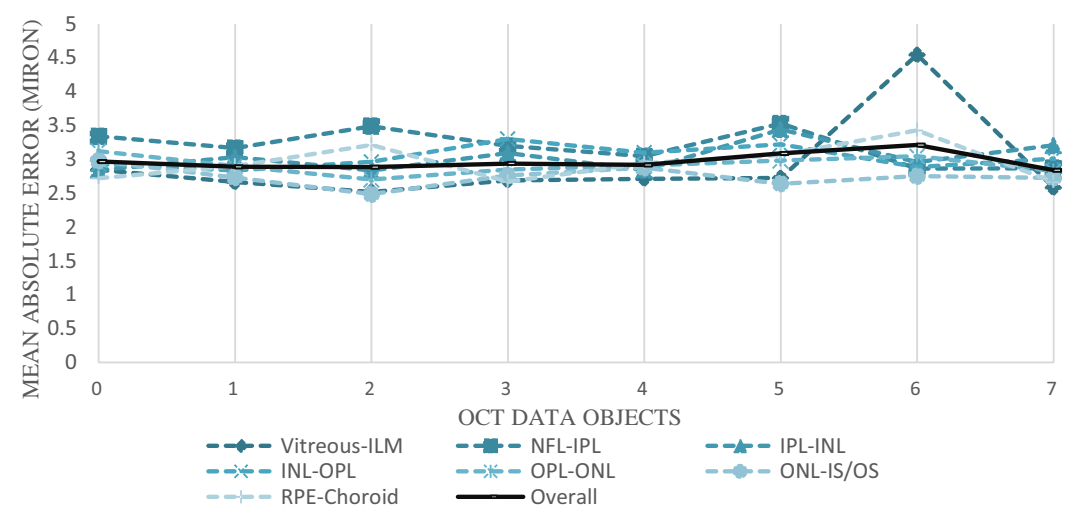

Fig. 20. Mean Absolute error of eight different OCT data objects. 

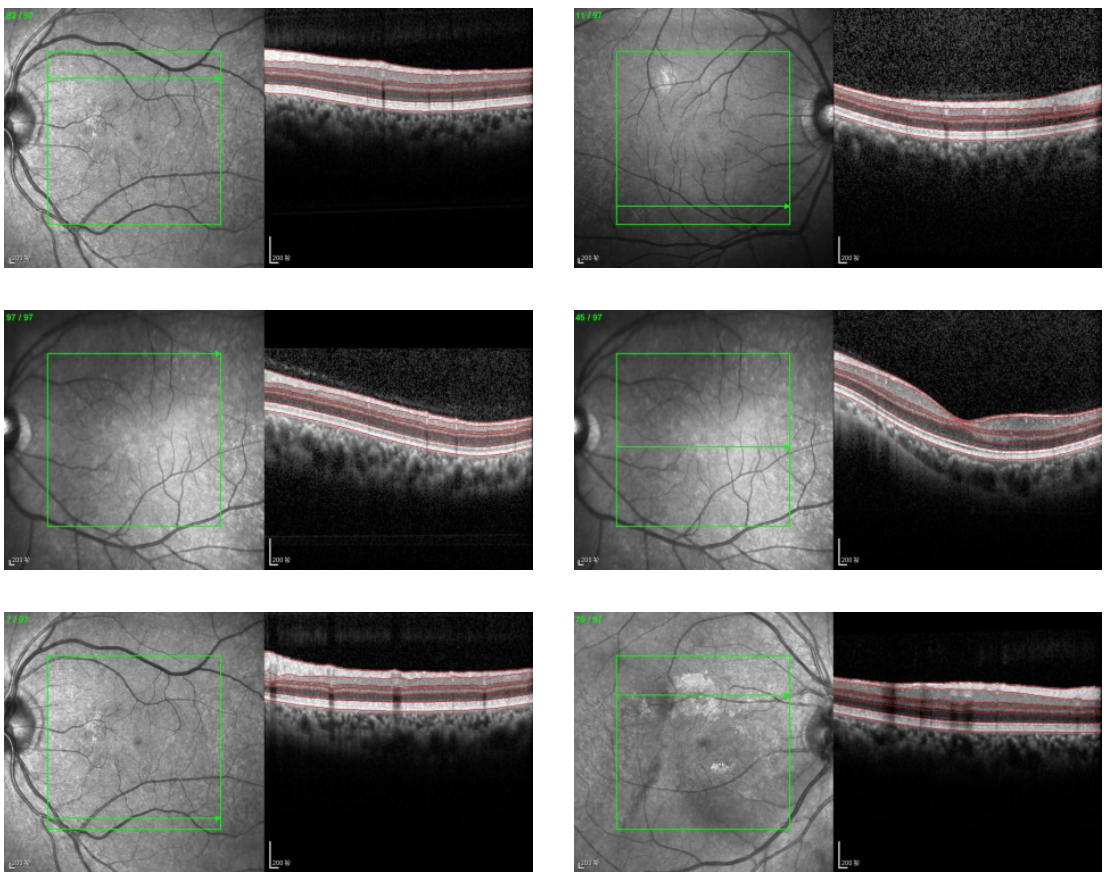

Fig. 21. Some experimental results from various imaging cases.

Some experimental results are shown in Fig. 19, with the images in the first row as the segmental results by our algorithm, and the images in the second row as the ones by OCTSeg. It can be seen from Fig. 19 that our segmental results is better than OCTSeg's visually. In addition, NFL-GCL boundary segmented by OCTSeg is very not accurate, and our method is more robust to images with shadow.

To show that our algorithm is reproducible, we separate the B-Scans into two groups according to its group number's parity. And calculated the absolute error and signed error of the two groups comparing to the average manual segmentation. See Table 4.

From Table 4, we can see that the absolute error difference between the odd group and even group is very small.

To further examine the stability of our algorithm, we calculated the absolute error for each OCT objects, and Fig. 20 shows that the overall absolute error is quite stable in these eight OCT objects which shows that our algorithm works well and stable in different datasets.

Figure 21 presents some experimental results for various cases. It can be seen that our automatic algorithm correctly segmented seven retinal layer boundary surfaces for retinal OCT images with large curvature, severe noise above the ILM, fovea, prominent vessels, or large shadows because of diseases. The average computation time was $3.5 \mathrm{~s}$ per frame (Intel Core I7 CPU at $3.0 \mathrm{GHz}$, and $8 \mathrm{~GB}$ RAM), and the program can be optimized to reduce the time further. Therefore, our algorithm is simple and fast.

Once the retinal layer segmentations have been performed successfully, every layer boundary surface can be visualized, and a thickness map between any two boundaries can be generated, which can be

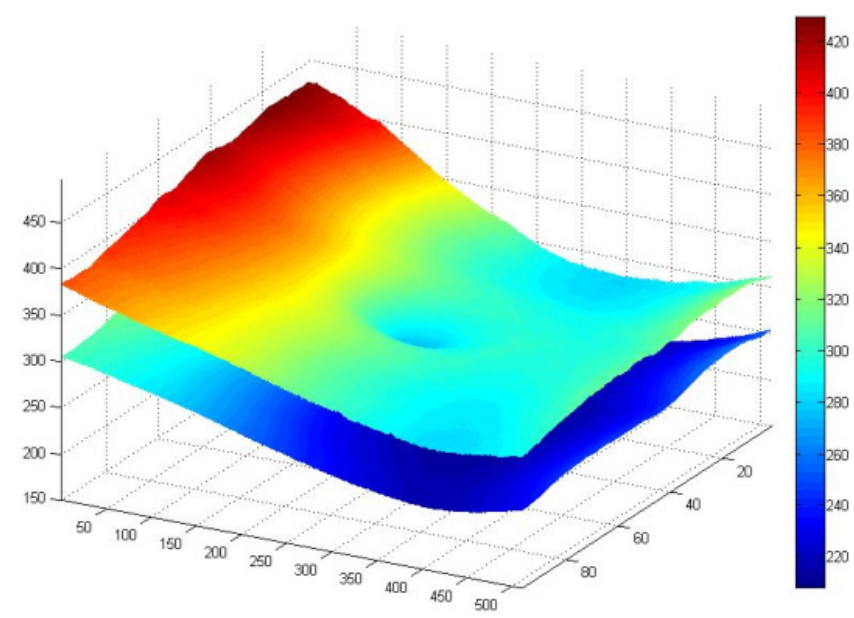

Fig. 22. Visualization of the Vitreous-ILM and RPE-Choroid boundary surfaces. 


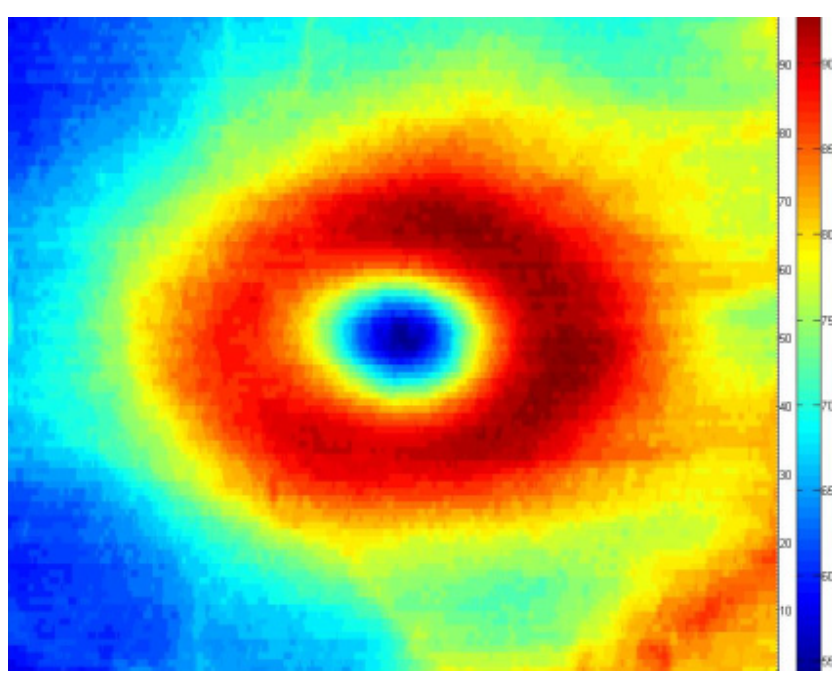

Fig. 23. Retinal thickness map determined from the VitreousILM and RPE-Choroid boundary surfaces.

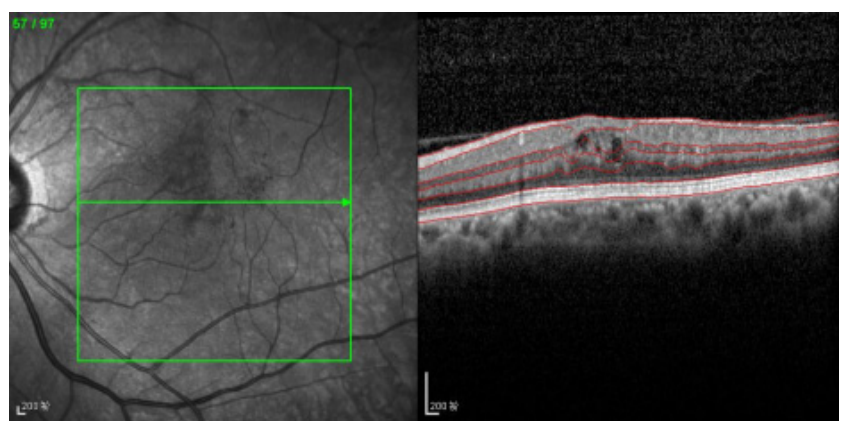

Fig. 24. Segmentation result for a retinal OCT image with serious abnormalities.

useful for disease diagnosis. An example visualization of the Vitreous-ILM and RPE-Choroid boundary surfaces is shown in Fig. 22, and the whole retinal thickness map determined from them is shown in Fig. 23.

As is the case for most of the current OCT image segmentation algorithms, our algorithm processes healthy or slightly abnormal retinal OCT images well but fails to accurately process retinal OCT images with serious diseases (Fig. 24).

\section{Conclusions}

We proposed a novel 3D segmentation method for retinal OCT volume data that uses pixel intensity, boundary position information, and intensity changes on both sides of the layer borders simultaneously. The method designs a specific 3D differential operator for the processing boundary to enhance the border, it conducts a 3D smoothing procedure to denoise the volume data, and it further utilizes the boundary position to produce an enhanced boundary volume data that serves as a better indicator for identifying the desired boundaries. Our method can segment seven prominent boundary surfaces, and it is automatic, efficient and practical. It should be pointed out that the mentioned strategy in our method should be applicable for other boundary surfaces, which would be our future work to do.

\section{Acknowledgments}

This research was supported by the National High Technology Research and Development Program of China ("863" Program) under Grant No. 2013AA013702 and the National Natural Science Foundation of China (No. 60971006).

\section{References}

1. D. C. Fernandez, H. M. Salinas, C. A. Puliafito, "Automated detection of retinal layer structures on optical coherence tomography images," Opt. Express 13(25), 10200-10216 (2005).

2. M. Mujat, R. C. Chan, B. Cense, B. H. Park, C. Joo, T. Akkin, T. C. Chen, J. F. de Boer, "Retinal nerve fiber layer thickness map determined from optical coherence tomography images," Opt. Express 13(23), 9480-9491 (2005).

3. H. Ishikawa, D. M. Stein, G. Wollstein, S. Beaton, J. G. Fujimoto, J. S. Schuman, "Macular segmentation with optical coherence tomography," Invest. Ophthalmol. Vis. Sci. 46(6), 2012-2017 (2005).

4. S. J. Chiu, X. T. Li, P. Nicholas, C. A. Toth, J. A. Izatt, S. Farsiu, "Automatic segmentation of seven retinal layers in SD-OCT images congruent with expert manual segmentation," Opt. Express 18(18), 19413-19428 (2010).

5. P. P. Srinivasan, S. J. Heflin, J. A. Izatt, V. Y. Arshavsky, S. Farsiu, "Automatic segmentation of up to ten layer boundaries in SD-OCT images of the mouse retina with and without missing layers due to pathology," Biomed. Opt. Express 5(2), 348-365 (2014).

6. F. LaRocca, S. J. Chiu, R. P. McNabb, A. N. Kuo, J. A. Izatt, S. Farsiu, "Robust automatic segmentation of corneal layer boundaries in SD-OCT images using graph theory and dynamic programming," Biomed. Opt. Express 2(6), 1524-1538 (2011).

7. S. Farsiu, S. J. Chiu, R. V. O'Connell, F. A. Folgar, E. Yuan, J. A. Izatt, C. A. Toth, "Quantitative 
Classification of Eyes with and without Intermediate Age-related Macular Degeneration Using Optical Coherence Tomography," Ophthalmology 121(1), 162-172 (2014).

8. S. J. Chiu, M. J. Allingham, P. S. Mettu, S. W. Cousins, J. A. Izatt, S. Farsiu, "Kernel regression based segmentation of optical coherence tomography images with diabetic macular edema," Biomed. Opt. Express 6(4), 1172-1194 (2015).

9. Q. Yang, D. C. K. Hood, K. Chan, C. A. Reisman et al., "Automated layer segmentation of macular OCT images using dual-scale gradient information," Opt. Express 18(20), 21293-21307 (2010).

10. Q. Yang, C. A. Reisman, K. Chan, R. Ramachandran, A. Raza, D. C. Hood, Automated segmentation of outer retinal layers in macular OCT images of patients with retinitis pigmentosa, Biomed. Opt. Express 9(2), 2493-2503 (2011).

11. A. Yazdanpanah, G. Hamarneh, B. R. Smith, M. V. Sarunic, "Segmentation of intra-retinal layers from optical coherence tomography images using an active contour approach," IEEE Trans. Med. Imaging 30(2), 484-496 (2011).

12. A. Mishra, A. Wong, K. Bizheva, D. A. Clausi, "Intra-retinal layer segmentation in optical coherence tomography images," Opt. Express 17(26), 23719-23728 (2009).

13. G. Itebeddine, R. Florence, I. Bloch, S. Tick, M. Paques, "Automated segmentation of macular layers in OCT images and quantitative evaluation of performances," Pattern Recognit. 44(8), 1590-1603 (2011).

14. A. R. Fuller, R. J. Zawadzki, S. Choi, D. F. Wiley, J. S. Werner, B. Hamann, "Segmentation of threedimensional retinal image data," IEEE Trans. Vis. Comput. Graphics 13(6), 1719-1726 (2007).

15. R. J. Zawadzki, A. R. Fuller, D. F. Wiley, B. Hamann et al., "Adaptation of a support vector machine algorithm for segmentation and visualization of retinal structures in volumetric optical coherence tomography data sets," J. Biomed. Opt. 12(4), 041206 (2007).

16. K. A. Vermeer, J. van der Schoot, H. G. Lemij, J. F. de Boer, "Automated segmentation by pixel classification of retinal layers in ophthalmic OCT images," Biomed. Opt. Express 2(6), 1743-1756 (2011).

17. A. Lang, A. Carass, M. Hauser, E. S. Sotirchos, P. A. Calabresi, H. S. Ying, J. L. Prince, "Retinal layer segmentation of macular OCT images using boundary classification," Biomed. Opt. Express 4(7), 1133-1152 (2013).

18. V. Kajić, B. Považay, B. Hermann, B. Hofer, D. Marshall, P. L. Rosin, W. Drexler, "Robust segmentation of intraretinal layers in the normal human fovea using a novel statistical model based on texture and shape analysis," Opt. Express 18(14), 14644-146 (2010).

19. M. K. Garvin, M. D. Abràmoff, R. Kardon, S. R. Russell et al., "Intraretinal layer segmentation of macular optical coherence tomography images using optimal 3-D graph search," IEEE Trans. Med. Imaging 10(10), 1495-1505 (2008).

20. M. K. Garvin, M. D. Abràmoff, X. Wu, S. R. Russell et al., "Automated 3-D intraretinal layer segmentation of macular spectral-domain optical coherence tomography Images," IEEE Trans. Med. Imaging 28(9), 1436-1447 (2009).

21. P. A. Dufour, L. Ceklic, H. Abdillahi, S. Schröder, S. De Dzanet, U. Wolf-Schnurrbusch, J. Kowal, "Graph-based multi-surface segmentation of OCT data using trained hard and soft constraints," IEEE Trans. Med. Imaging 32(3), 531-543 (2013).

22. K. Lee, M. Niemeijer, M. K. Garvin, Y. H. Kwon, M. Sonka, M. D. Abràmoff, "Segmentation of the optic disc in 3-D OCT scans of the optic nerve head," IEEE Trans. Med. Imaging 29(1), 159-168 (2010).

23. K. Raheleh, H. Rabbani, M. D. Abramoff, M. Sonka, "Intra-retinal layer segmentation of 3D optical coherence tomography using coarse grained diffusion map," Med. Image Anal 17, 907-928 (2013).

24. H. Bogunovic, M. Sonka, Y. H. Kwon, P. Kemp, M. D. Abramoff, Wu Xiaodong, "Multi-surface and multi-field co-segmentation of 3 -D retinal optical coherence tomography," IEEE Trans. Med. Imaging 33(12), 2242-2253 (2014).

25. F. Shi, X. Chen, H. Zhao, W. Zhu, D. Xiang, E. Gao, M. Sonka, H. Chen. "Automated 3-D retinal layer segmentation of macular optical coherence tomography images with serous pigment epithelial detachments," IEEE Trans. Med. Imaging 34(2), 441-452 (2015).

26. J. Tian, B. Varga, G. M. Somfai, W.-H. Lee, W. E. Smiddy, D. Cabrera DeBuc, "Real-time automatic segmentation of optical coherence tomography volume data of the macular region," PLOS ONE 10(8), e0133908 (2015). doi:10.1371/journal.pone.0133908.

27. T. Fabritius, S. Makita, M. Miura, M. Risto, Y. Yoshiaki, "Automated segmentation of the macula by optical coherence tomography," Opt. Express, 17(18), 15659 (2009).

28. S. Niu, Q. Chen, L. de Sisternes, D. L. Rubin, W. Zhang, Q. Liu," Automated retinal layers segmentation in SD-OCT images using dual-gradient and spatial correlation smoothness constraint," Comput. Biol. Med. 54, 116-128 (2014).

29. Y. Xin, R. C. Jennifer, R. K. Wang, "User-guided segmentation for volumetric retinal optical coherence tomography images," J. Biomed. Opt. 19(8), e086020 (2014). doi:10.1117/1.JBO.19.8.086020. 
30. R. Kafieh, H. Rabbani, I. Selesnick, "Three dimensional data-driven multi scale atomic representation of optical coherence tomography," IEEE Trans. Med. Imaging 34(5), 1042-1062 (2015).

31. H. Chen, X. Chen, Z. Qiu, D. Xiang, W. Chen, F. Shi, J. Zheng, W. Zhu, M. Sonka, "Quantitative analysis of retinal layers' optical intensities on 3D optical coherence tomography for central retinal artery occlusion," Scientific Reports, Scientific Reports 5, Article number: 9269 (2015).

32. C. Wang, Y. Wang, D. Kaba, H. Zhu, Y. Lv, Z. Wang, X. Liu, Y. Li, "Segmentation of intra- retinal layers in 3D optic nerve head images," Image and Graphics: 8th International Conference, ICIG 2015, Tianjin, China, August 13-16 2015, Proceedings, Lecture Notes in Computer Science, Springer, pp. 321-332, (2015).

33. C. Wang, Y. Wang, D. Kaba, Z. Wang, X. Liu, $\mathrm{Y}$. Li, "Automated layer segmentation of $3 \mathrm{D}$ macular images using hybrid methods," Image and Graphics: 8th International Conference, ICIG 2015, Tianjin, China, August 13-16 2015, Proceedings, Lecture Notes in Computer Science, Springer, pp. 614-628, (2015). 